\title{
Effect of hydroxyurea on the intracellular multiplication of Toxoplasma gondii, Leishmania amazonensis and Trypanosoma cruzi
}

E.J.T. Melo and H.J. Beiral
Laboratório de Biologia Celular, Centro de Biociências e Biotecnologia,

Universidade Estadual do Norte Fluminense, Campos dos Goytacazes, RJ, Brasil

\author{
Correspondence \\ E.J.T. Melo \\ Laboratório de Biologia Celular \\ CBB, Universidade Estadual do \\ Norte Fluminense \\ Av. Alberto Lamego, 2000 \\ 28013-600 Campos dos Goytacazes, RJ \\ Brasil \\ Fax: +55-022-2726-1514 \\ E-mail: ejtm@uenf.br
}

Received May 29, 2001

Accepted October 14, 2002

\begin{abstract}
Toxoplasma gondii, Leishmania amazonensis and Trypanosoma cruzi are obligate intracellular parasites that multiply until lysis of host cells. The present study was undertaken to evaluate the effect of hydroxyurea (an inhibitor of cell division at the G1/S phase) on the multiplication of L. amazonensis, T. gondii, and T. cruzi in infected host cells. Infected cells were treated with hydroxyurea $(4 \mathrm{mM})$ for 48 h. Hydroxyurea arrested intracellular multiplication of all infective forms of the parasites tested. In treated cultures, the percent of infected host cells decreased (50-97\%) and most intracellular parasites were eliminated. Ultrastructural observations showed no morphologic change in host cells while intracellular parasites presented drastic morphologic alterations or disruption. The results strongly suggest that hydroxyurea was able to interfere with the multiplication of intracellular parasites, leading to an irreversible morphological effect on L. amazonensis, T. gondii, and T. cruzi without affecting the host cells.
\end{abstract}

Key words

- Toxoplasma gondii

- Trypanosoma cruzi

- Leishmania amazonensis

- Hydroxyurea
The protozoan parasites Toxoplasma gondii, Leishmania amazonensis and Trypanosoma cruzi are etiologic agents that cause toxoplasmosis, leishmaniasis and Chagas' disease, respectively (1-3). These parasites invade the host while being localized within a vacuole known as the parasitophorous vacuole (PV). L. amazonensis and T. gondii develop within the PV whereas $T$. cruzi escapes to the cytoplasm of host cells. It is important to point out that the latter multiplies inside the cell cytoplasm and is released into the intercellular space after rupture of the host cell (4). In experimental animals, control of infection with therapeutic drugs generally causes drastic effects on the host cells (5-7). Hydroxyurea is a drug that specifically inhibits the cell cycle in the G1/S phase inactivating the enzyme ribonucleotide reductase and the synthesis of DNA (8). Different concentrations of hydroxyurea have been used for the treatment of several types of cancer $(8,9)$ and various concentrations have also been tested against trypomastigotes and epimastigotes of $T$. cruzi, promastigotes of Crithidia luciliae (5-20 mM) (10), tachyzoites of $T$. gondii (4 mM) (11), CD4 cell infected with HIV $(100-200 \mathrm{mg} / \mathrm{kg})$ and $T r i-$ chomonas vaginalis $(5 \mathrm{mM})(12)$. However, hydroxyurea at a concentration up to $5 \mathrm{mM}$ 
has been used to synchronize the cell and parasite cultures without causing toxic effects $(10,12)$. We have recently shown that hydroxyurea, an inhibitor of DNA synthesis, inhibited the intracellular multiplication of $T$. gondii followed by tachyzoite elimination (11). We have extended this study by also using hydroxyurea against the intracellular parasites L. amazonensis and T. cruzi.

Macrophages were collected from Swiss mice and cultivated for $24 \mathrm{~h}$ in medium 199 supplemented with fetal calf serum (FCS) at $37^{\circ} \mathrm{C}$ under a continuous flow of $5 \% \mathrm{CO}_{2}$ (13). L. amazonensis (MHUM/Br/175/Josefa strain) was cultivated for 7 days at $25^{\circ} \mathrm{C}$. The culture was infected for $1 \mathrm{~h}$, washed with sodium phosphate-buffered saline (PBS) to remove extracellular parasites, and incubated for $72 \mathrm{~h}$ in medium 199 at $37^{\circ} \mathrm{C}$ under a continuous flow of $5 \% \mathrm{CO}_{2}$. After this procedure, most cells were infected. For infection with T. cruzi and T. gondii, Vero cells (kidney fibroblasts from African green monkeys) were obtained through periodic passages and used when semi-confluent on Falcon plates or in flask cultures (11). The cultures were infected with $T$. cruzi trypomastigotes ( $\mathrm{Y}$ strain obtained from a culture of Vero cells infected for $6-7$ days at $37^{\circ} \mathrm{C}$ )

Figure 1. Effect of hydroxyurea on cultures of host cells infected with L. amazonensis, T. gondii, and T. cruzi. $A$, In the untreated culture, most host cells were infected (filled columns). After treatment of infected cells with $4 \mathrm{mM}$ hydroxyurea for $48 \mathrm{~h}$, the percent of infected cells was significantly decreased (open columns). $B$, Mean number of intracellular parasites in infected host cells. The number of intracellular parasites increased in untreated cells (filled columns) and was significantly decreased in treated cells (open columns) after incubation with $4 \mathrm{mM}$ hydroxyurea for $48 \mathrm{~h}$. Data are reported as means \pm SD of four independent experiments. ${ }^{*} P<0.05$ compared to untreated cells (Student $t$-test). presence of medium 199 supplemented with $5 \%$ FCS $(11,13)$. All parasites were assumed to have interacted with the cells because the parasite:host cell relationship was 10:1.

Hydroxyurea (Merck Chemical Co., Darmstadt, Germany) was dissolved in PBS (4 $\mathrm{M}$ stock solution) and the solution was mixed with medium 199 in cultured cells at a final concentration of $4 \mathrm{mM}$. This drug concentration has been established for synchronization of the cell cycle, and has no toxic effects on the cells (10-13). The drug was added to the infected cells during intense parasite proliferation. After incubation with hydroxyurea, the cultures were washed with PBS, fixed with Bouin's fixative, stained with Giemsa and observed under a light microscope (63X objective Axioplan, Zeiss, Jena, Germany). The percentage of infected cells and the mean number of intracellular parasites were determined by examination of at least 400 cells.

Statistical analysis was carried out using the Student $t$-test, with the level of significance set at $\mathrm{P}<0.5$. The data shown are representative of five experiments in triplicate.

The cultures in flasks were infected with parasites and incubated with the drug as described above for transmission electron microscopy. The cultures were fixed for 60 min at room temperature in a solution containing 1\% glutaraldehyde, $4 \%$ paraformaldehyde, $5 \mathrm{mM} \mathrm{CaCl}_{2}$ and $5 \%$ sucrose in 0.1 $\mathrm{M}$ cacodylate buffer, $\mathrm{pH}$ 7.2. Cells were post-fixed for $1 \mathrm{~h}$ in a solution containing $1 \%$ osmium tetroxide, $0.8 \%$ potassium ferrocyanide and $5 \mathrm{mM} \mathrm{CaCl}_{2}$ in $0.1 \mathrm{M}$ cacodylate buffer, $\mathrm{pH}$ 7.2. The cells were rinsed in $0.1 \mathrm{M}$ cacodylate buffer, dehydrated in acetone and embedded in Epon. Thin sections were stained with uranyl acetate and lead citrate and observed under a Zeiss 900 transmission electron microscope.

When infected cells were incubated with $4 \mathrm{mM}$ hydroxyurea for $48 \mathrm{~h}$, the number of infected cells decreased significantly in all treated cultures (Figure 1A, open columns) 
when compared with untreated control cells (Figure 1A, filled columns). A similar significant decrease was observed in the mean number of intracellular parasites in the three cultures (Figure 1B, open columns) when compared with control untreated cells (Figure $1 \mathrm{~B}$, filled columns). A drastic reduction in the number of infected cells was also observed in host cells infected with $T$. gondii. These results clearly show that the presence of hydroxyurea reduced both the percentage of infected cells and the number of intracellular parasites.

Light and electron microscopy was used to observe the effects of the drug on parasite morphology and ultrastructure. Tachyzoites are elongated and curved, while amastigotes of $T$. cruzi and L. amazonensis have a spherical shape. Light microscopy demonstrated that treated Vero cells infected with $T$. gondii showed normal morphology, although intracellular parasites, when present, were dis-

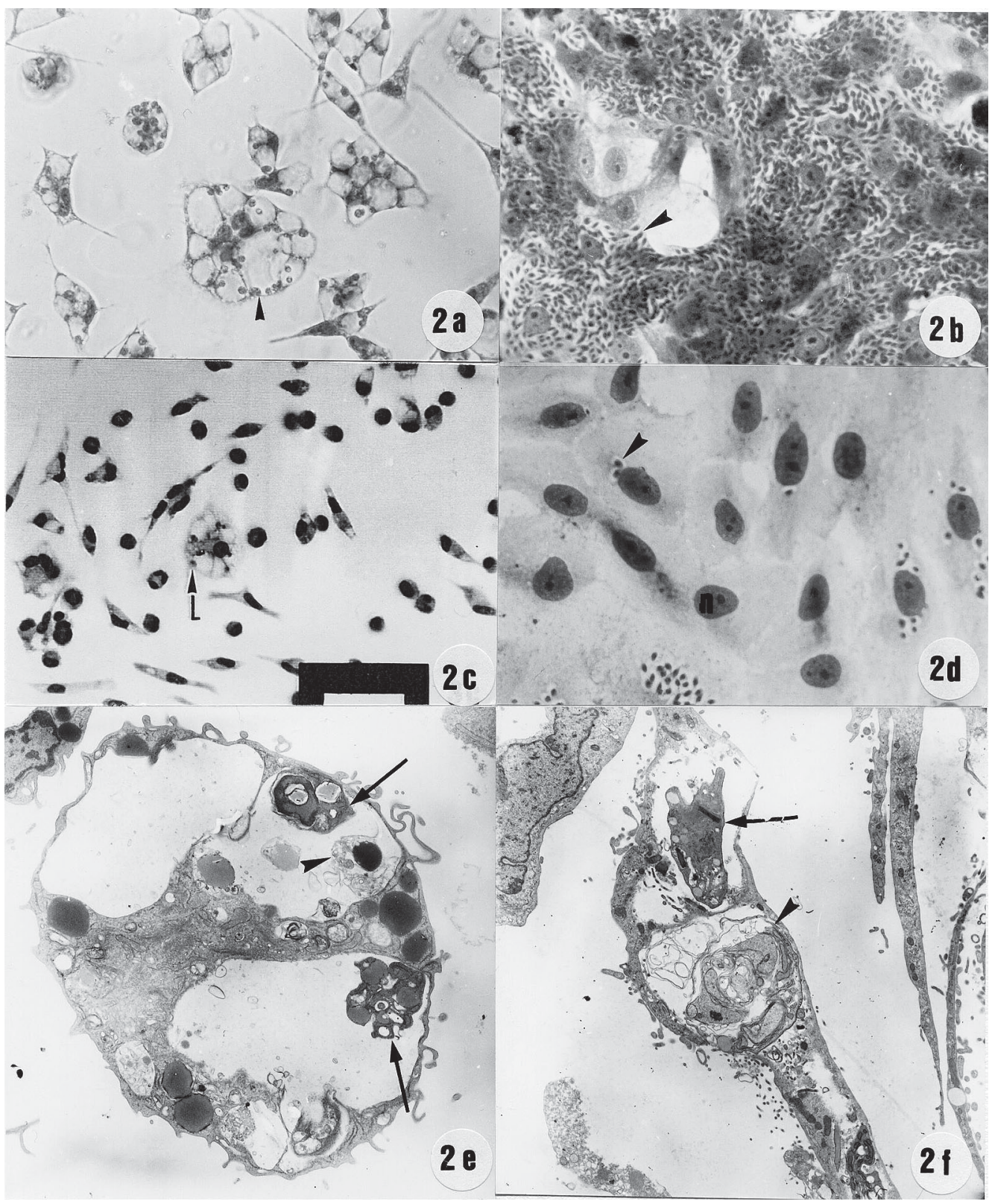

Figure 2. Effect of hydroxyurea on the intracellular multiplication of $T$. gondii, L. amazonensis and T. cruzi. a-d, Giemsa-stained host cells infected with parasites. Macrophages infected with L. amazonensis (a) or Vero cells infected with $T$. cruzi (b) were treated $(c, d)$ with $4 \mathrm{mM}$ hydroxyurea for $48 \mathrm{~h}$. A large number of parasites are observed in the cytoplasm of untreated cultures $(a, b$, arrowhead). After incubation for $48 \mathrm{~h}$ in the presence of $4 \mathrm{mM}$ hydroxyurea, very few cells were infected with L. amazonensis (c, arrowhead) or T. cruzi (d, arrowhead). e-f, Ultrastructural observation of macrophages or Vero cells infected with $L$. amazonensis (e) and T. cruzi (f), and treated with $4 \mathrm{mM}$ hydroxyurea for $48 \mathrm{~h}$. After incubation with 4 $\mathrm{mM}$ hydroxyurea, amastigotes of $L$. amazonensis with morphological changes (arrow) or disrupted (arrowhead) were observed. Vero cells infected with $T$. cruzi showed intracellular parasites with morphological alterations (arrow) or disrupted (arrowhead) in the cytoplasm of host cells. $L=L$. amazonensis, $n=$ nucleus. Panels $a-d=900 X$ panels e $f=15,000 X$ 
rupted (data not shown), in agreement with earlier observations (11). Culture of macrophages infected with L. amazonensis showed intense parasite proliferation (Figure 2a, arrowhead). When infected macrophages were treated with hydroxyurea, a larger number of uninfected cells with normal morphology was observed (Figure 2c), while intravacuolar parasites, present in some infected cells, did not proliferate (Figure 2c, arrowhead). Ultrastructural aspects of parasites showed the typical eukaryotic organelles and nucleus. When infected macrophages were observed by transmission electron microscopy, proliferating amastigotes were observed in the PV (data not shown). Treated infected cells demonstrated intravacuolar amastigotes with drastic ultrastructural alterations (Figure 2e, arrow) or disrupted (Figure 2e, arrowhead). The cytoplasm of the organelles in the host cells did not present any alterations (data not shown). Intense $T$. cruzi multiplication was observed inside the cytoplasm of Vero cells (Figure 2b). When infected cells were treated with hydroxyurea, most uninfected cells presented normal morphology (Figure 2d), and the intracellular parasites were not proliferating (Figure 2d, arrowhead). When observed by transmission electron microscopy, the intracellular parasites presented ultrastructural alterations (Figure 2f, arrow) or were disrupted ( $2 \mathrm{f}$, arrowhead). These results suggest that host cells treated with hydroxyurea were preserved and intracellular parasites showed an arrested cell cycle or were disrupted.

T. gondii, L. amazonensis and T. cruzi multiply within host cells, with parasite division occurring at different times. This fact can result in a variation of the mean number of intracellular parasites destroyed during incubation with the drug (Figure 1B). The effect of hydroxyurea is specific for the cell cycle and some studies have demonstrated that hydroxyurea used in synchronization experiments does not produce morphological or ultrastructural alterations in treated cells $(10,12,14,15)$. Hydroxyurea arrests DNA replications without interfering with the $\mathrm{S}$ phase. In fact, DNA synthesis is a process that occurs independently of other aspects of cell growth. For this reason, hydroxyurea has been used in cell synchronization studies and for cancer treatment (16). Recently, several hydroxyurea concentrations (5-20 mM) were tested on T. cruzi demonstrating that, although the drug inhibited DNA synthesis in the nucleus and kinetoplast, other metabolic processes in the cell were not affected (10).

The present results demonstrated that treatment with hydroxyurea for $48 \mathrm{~h}$ arrested parasite multiplication, causing drastic morphologic or ultrastructural alterations or complete elimination of the parasites. During incubation with the drug, the host cell defense may be activated or become more effective against these parasites. This possibility has already been investigated in our laboratory. Studies of the in vivo action of hydroxyurea against infection with different parasites are also currently being conducted in our laboratory.

\section{References}

1. Morisaki JH, Heuser JE \& Sibley LD (1995). Invasion of Toxoplasma gondii occurs by active penetration of the host cell. Journal of Cell Science, 108: 2457-2464

2. Araújo-Jorge TC (1989). The biology of Trypanosoma cruzi-macrophage interaction. Memórias do Instituto Oswaldo Cruz, 84: 441462.

3. Lang T, Helio R, Kaye PM \& Antoine J-C (1994). Leishmania donovani-infected macrophages: characterization of the parasitophorous vacuole and potential role of this organelle in antigen presentation. Journal of Cell Biology, 107: 2137-2150.

4. Mauel J (1996). Intracellular survival of protozoan parasites with special reference to Leishmania spp., Toxoplasma gondii and Trypanosoma cruzi. Advances in Parasitology, 38: 1-51.

5. Gormley PD, Pavesio CE, Minnasian D \& Lightman S (1998). Effects of drug therapy on Toxoplasma cysts in an animal model of acute and chronic disease. Investigative Ophthalmology and Visual Sci- 
ence, 39: 1171-1175.

6. Brener Z, Tafuri WL \& Almeida Maria T (1969). An electron microscope study of Trypanosoma cruzi intracellular forms in mice treated with an active nitrofuran compound. Revista do Instituto de Medicina Tropical de São Paulo, 11: 245-249.

7. Teixeira ARL, Silva R, Cunha Neto E, Santana JM \& Rizzo LV (1990). Malignant non-Hodgkin's lymphomas in Trypanosoma cruzi-infected rabbits treated with nitroagents. Journal of Comparative Pathology, 103: 37-48.

8. Yarbro JW (1992). Mechanism of action of hydroxyurea. Seminars in Oncology, 19: 1-10.

9. Melo EJT, Mayerhoffer RO \& De Souza W (2000). Hydroxyurea inhibits intracellular Toxoplasma gondii multiplication. FEMS Microbiology Letters, 185: 79-82.

10. Galanti N, Dvorak JA, Grenet J \& McDaniel JP (1994). Hydroxyureainduced synchrony of DNA replication in the Kinetoplastida. Experimental Cell Research, 214: 225-230.

11. Simonelli C, Nasti G, Vaccher E, Tirelli U, Zanussi S, Depoali P,
Comar M \& Giacca M (1996). Hydroxyurea treatment in HIV-infected patients. Journal of Acquired Immune Deficiency Syndromes and Human Retrovirology, 13: 462-464.

12. Benchimol M (1999). Hydrogenosome autophagy: an ultrastructural and cytochemical study. Biology of the Cell, 91: 165-174.

13. Carvalho TMU \& De Souza W (1989). Early events related with the behaviour of Trypanosoma cruzi within an endocytic vacuole in mouse peritoneal macrophages. Cell Structure and Function, 14: 383-392.

14. Hofer A, Ekaren JT \& Thelander L (1998). Allosteric regulation of Trypanosoma brucei ribonucleotide reductase studied in vitro and in vivo. Journal of Biological Chemistry, 273: 34098-34104.

15. Taylor WR, Agarwal ML, Agarwal A, Stacey DW \& Stark GR (1999). P53 inhibits entry into mitosis when DNA synthesis is blocked. Oncogene, 18: 283-295.

16. Gwilt PR \& Tracewell WG (1998). Pharmacokinetics and pharmacodynamics of hydroxyurea. Clinical Pharmacokinetics, 34: 347-358. 